\title{
Schrödinger wave functional in quantum Yang-Mills theory from precanonical quantization
}

\author{
Igor V. Kanatchikov \\ School of Physics and Astronomy \\ University of St Andrews, St Andrews KY16 9SS, UK \\ E-mail: ik25@st-andrews.ac.uk \\ Quantum Information Center in Gdańsk (KCIK), \\ 81-831 Sopot, Poland
}

\begin{abstract}
A relation between the precanonical quantization of pure Yang-Mills fields and the standard functional Schrödinger representation in the temporal gauge is discussed. It is shown that the latter can be obtained from the former when the ultraviolet parameter $\varkappa$ introduced in precanonical quantization goes to infinity. In this limiting case, the Schrödinger wave functional can be expressed as the trace of the Volterra product integral of Clifford-algebra-valued precanonical wave functions restricted to a field configuration, and the canonical functional derivative Schrödinger equation together with the quantum Gauß constraint can be derived from the Dirac-like precanonical Schrödinger equation.
\end{abstract}

Key words: Quantum Yang-Mills theory, De Donder-Weyl formalism, precanonical quantization, canonical quantization, functional Schrödinger representation, Gauss constraint, Clifford algebra, Volterra product integral.

\section{Introduction}

The canonical quantization of YM field theory in the functional Schrödinger picture of QFT [1 13] leads (in the temporal gauge $A_{0}^{a}(\mathbf{x})=0$ ) to the description of the corresponding quantum field in terms of the Schrödinger wave functional $\Psi\left(\left[A_{i}^{a}(\mathrm{x})\right], t\right)$ which satisfies the functional derivative Schrödinger equation

$$
\mathrm{i} \hbar \partial_{t} \mathbf{\Psi}=\int d \mathbf{x}\left(-\frac{\hbar^{2}}{2} \frac{\delta}{\delta A_{a}^{i}(\mathbf{x})} \frac{\delta}{\delta A_{a}^{i}(\mathbf{x})}+\frac{1}{4} F_{a}{ }^{i j}(\mathbf{x}) F_{a}{ }^{i j}(\mathbf{x})\right) \boldsymbol{\Psi}
$$

and the Gauß law constraint on the physical states

$$
\left(\partial_{i} \frac{\delta}{\delta A_{i}^{a}(\mathbf{x})}+g C^{a}{ }_{b c} A_{i}^{b}(\mathbf{x}) \frac{\delta}{\delta A_{i}^{c}(\mathbf{x})}\right) \Psi=0
$$

which picks the gauge invariant functionals $\Psi$. 
Precanonical quantization of YM fields proposed in [14,15] leads to the description in terms of the Clifford-algebra-valued wave function $\Psi\left(A_{\mu}^{a}, x^{\mu}\right)$ which satisfies the partial derivative precanonical Schrödinger equation

$$
\mathrm{i} \hbar \gamma^{\mu} \partial_{\mu} \Psi=\left(\frac{1}{2} \hbar^{2} \varkappa \frac{\partial^{2}}{\partial A_{a}^{\mu} \partial A_{\mu}^{a}}-\frac{1}{2} \mathrm{i} g \hbar C^{a}{ }_{b c} A_{\mu}^{b} A_{\nu}^{c} \gamma^{\nu} \frac{\partial}{\partial A_{\mu}^{a}}\right) \Psi=: \frac{1}{\varkappa} \widehat{H} \Psi
$$

and the constraint

$$
\widehat{\pi}_{a}^{(\nu \mu)} \Psi=-\mathrm{i} \hbar \varkappa\left(\gamma^{\mu} \frac{\partial}{\partial A_{\nu}^{a}}+\gamma^{\nu} \frac{\partial}{\partial A_{\mu}^{a}}\right) \Psi \approx 0
$$

which is the quantum counterpart of the antisymmetry of the classical YM field strength

$$
F_{\mu \nu}^{a}:=\partial_{\mu} A_{\nu}^{a}-\partial_{\nu} A_{\mu}^{a}+g C^{a}{ }_{b c} A_{\mu}^{b} A_{\nu}^{c} .
$$

The notations and conventions used throughout this paper follow [14,15].

In precanonical quantization [15-18, one introduces an ultraviolet parameter $\varkappa$ of dimension of the inverse spatial volume. One of the appearances of this parameter is in the representation of the differential form associated with an infinitesimal spatial volume element $\mathrm{d} \mathbf{x}$ as an element of the Clifford algebra of the corresponding $n$-dimensional pseudo-Euclidean space-time $\mathbb{R}^{1, n-1}$ (our signature convention is $+---\ldots$ )

$$
\mathrm{d} \mathbf{x}:=\mathrm{d} x^{1} \wedge \mathrm{d} x^{2} \wedge \ldots \wedge \mathrm{d} x^{n-1} \mapsto \frac{1}{\varkappa} \gamma_{0} .
$$

In what follows, we use the standard notation $\gamma_{0}=\beta$ with $\beta^{2}=1$ and set $\hbar=1$ for convenience.

In this paper, we show that the description of quantum YM fields in the functional Schrödinger picture of QFT obtained from canonical quantization follows from the quantum YM theory - obtained by precanonical quantization - in the singular limiting case corresponding to the infinite value of the ultraviolet parameter $\varkappa$ introduced by precanonical quantization.

From the point of view of standard QFT, at first glance, the claim that (1.3) may have something to do with (1.1) sounds improbable at least. Nevertheless, in [20, 21], improving an earlier consideration in [22], we have already shown that, in the case of interacting scalar fields, the description of quantum fields in the functional Schrödinger picture can be derived from the precanonical description in the limiting case corresponding to the inversion of the map (1.6), i.e. when $\frac{1}{\varkappa}$ is infinitesimal. In this paper, we will show that a similar relation exists between the functional Schrödinger equation and the precanonical Schrödinger equation for quantum pure YM field. The insights from [20,21] will be closely followed in the present consideration.

Intuitively, the relation between the Schrödinger wave functional and the precanonical wave function is based on the probabilistic interpretation of the former, as the probability amplitude of finding a field configuration $A(\mathbf{x})$ at some moment of time $t$, and the latter, as the probability amplitude of observing the field value $A$ at the space-time point $x$, that allows us to expect that the time dependent complex functional probability amplitude $\boldsymbol{\Psi}([A(\mathbf{x})], t)$ is a composition of space-time dependent Clifford-valued probability amplitudes given by the precanonical wave function $\Psi(A, x)$. 
Let us recall that precanonical quantization [16-19] is based on the De DonderWeyl (DW) Hamiltonian theory [23] which treats space-time variables on equal footing. In this formulation, the Poisson brackets are defined on differential forms representing the dynamical variables, that leads to the Poisson-Gerstenhaber algebra structure generalizing the Poisson algebra in the canonical Hamiltonian formalism [19, 24] (see also [25-27]). The result of its quantization (a small Heisenberg-like subalgebra of it) is that both the operators and wave functions are Clifford-algebra-valued, and the precanonical Schrödinger equation includes the space-time Dirac operator instead of the standard time derivative. One of the striking features of this formulation of quantized fields is that, as a consequence of the evolution of precanonical wave function given by the precanonical Schrödinger equation, it allows us to reproduce the classical field equations as the equations of expectation values of operators defined by precanonical quantization, thus generalizing the Ehrenfest theorem [15. Moreover, by treating the space-time variables on equal footing, the precanonical quantization approach provides a very natural and promising framework for quantization of gravity [28, 29].

Other recent discussions of gauge fields and gravity from the point of view of classical DW Hamiltonian theory and its geometrizations can be found in [30 36].

Most recently, the consideration of the eigenvalue problem for the DW Hamiltonian operator $\widehat{H}$ on the right-hand side of (1.3) has allowed us to estimate the non-vanishing gap in the spectrum of quantum $\mathrm{SU}(2)$ pure YM theory on $\mathbb{R}^{n}$ obtained from the precanonical quantization [37. This paper, by establishing a connection of precanonical approach with the standard formulation of quantum YM theory in the functional Schrödinger representation, can be viewed as an additional substantiation of the identification of the gap in the spectrum of precanonical DW Hamiltonian operator with the mass gap in the energy spectrum of the canonical Hamiltonian operator.

\section{From precanonical wave function to the Schrödinger wave functional}

Our task is to investigate a connection between the functional Schrödinger representation of quantum YM theory and precanonical quantization. In the case of interacting scalar fields this connection was established in Refs. [20,21] which refine an earlier treatment in [22. Similarly, our current treatment can be seen as an improvement of our earlier discussion in [14] which was based on [22].

A possible existence of a relation between the functional Schrödinger picture and the precanonical descripton implies that the Schrödinger wave functional $\mathbf{\Psi}([A(\mathbf{x})], t)$ can be expressed as a functional of precanonical wave functions restricted to a specific field configuration $\Sigma: A=A(\mathbf{x})$ at time $t$ :

$$
\boldsymbol{\Psi}([A(\mathrm{x})], t)=\boldsymbol{\Psi}\left(\left[\Psi_{\Sigma}(\mathrm{x}, t), A(\mathrm{x})\right]\right)
$$

where we have denoted the restriction of precanonical wave function $\Psi(A, x)$ to $\Sigma$ as $\Psi_{\Sigma}(\mathbf{x}, t):=\Psi(A=A(\mathbf{x}), \mathbf{x}, t)$. Then the time derivative of $\Psi$ is obtained by the chain rule differentiation

$$
\mathrm{i} \partial_{t} \Psi=\operatorname{Tr} \int d \mathbf{x}\left\{\frac{\delta \Psi}{\delta \Psi_{\Sigma}^{T}(\mathbf{x}, t)} \mathrm{i} \partial_{t} \Psi_{\Sigma}(\mathbf{x}, t)\right\}
$$


where $\Psi^{T}$ is the transpose matrix of $\Psi$. In what follows, for brevity, we will denote $\Psi_{\Sigma}(\mathbf{x}, t)$ simply as $\Psi_{\Sigma}(\mathbf{x})$ or even $\Psi_{\Sigma}$ when appropriate.

The first and the second total variational derivatives of $\Psi$ with respect to $A_{\mu}^{a}(\mathbf{x})$ (denoted as $\left.\frac{\boldsymbol{\delta}}{\boldsymbol{\delta} A_{\mu}^{a}(\mathbf{x})}\right)$ take the form

$$
\begin{aligned}
\frac{\boldsymbol{\delta} \Psi}{\boldsymbol{\delta} A_{\mu}^{a}(\mathbf{x})} & =\frac{\bar{\delta} \boldsymbol{\Psi}}{\bar{\delta} A_{\mu}^{a}(\mathbf{x})}+\operatorname{Tr}\left\{\frac{\delta \Psi}{\delta \Psi_{\Sigma}^{T}(\mathbf{x})} \partial_{A_{\mu}^{a}} \Psi_{\Sigma}(\mathbf{x})\right\} \\
\frac{\boldsymbol{\delta}^{2} \boldsymbol{\Psi}}{\boldsymbol{\delta} A_{\mu}^{a}(\mathbf{x}) \boldsymbol{\delta} A^{a \mu}(\mathbf{x})} & =\frac{\bar{\delta}^{2} \boldsymbol{\Psi}}{\bar{\delta} A_{\nu}^{a}(\mathbf{x}) \bar{\delta} A^{a \nu}(\mathbf{x})}+\operatorname{Tr}\left\{\frac{\delta \boldsymbol{\Psi}}{\delta \Psi_{\Sigma}^{T}(\mathbf{x})} \delta(\mathbf{0}) \partial_{A_{\mu}^{a}} \partial_{A^{a \mu}} \Psi_{\Sigma}(\mathbf{x})\right\} \\
& +\operatorname{Tr} \operatorname{Tr}\left\{\frac{\delta^{2} \boldsymbol{\Psi}}{\delta \Psi_{\Sigma}^{T}(\mathbf{x}) \otimes \delta \Psi_{\Sigma}^{T}(\mathbf{x})} \partial_{A_{\mu}^{a}} \Psi_{\Sigma}(\mathbf{x}) \otimes \partial_{A^{a \mu}} \Psi_{\Sigma}(\mathbf{x})\right\} \\
& +2 \operatorname{Tr}\left\{\frac{\delta \bar{\delta} \mathbf{\Psi}}{\delta \Psi_{\Sigma}^{T}(\mathbf{x}) \bar{\delta} A^{a \nu}(\mathbf{x})} \partial_{A_{\nu}^{a}} \Psi_{\Sigma}(\mathbf{x})\right\}
\end{aligned}
$$

where $\frac{\bar{\delta}}{\delta A_{\mu}^{a}(\mathbf{x})}$ denotes the partial functional derivative with respect to $A_{\mu}^{a}(\mathbf{x}), \partial_{A_{\mu}^{a}}$ is the same as $\frac{\partial}{\partial A_{\mu}^{a}}$ and $\delta(\mathbf{0})$ is the $(n-1)$-dimensional delta-function at $\mathbf{x}=0$ which results from the variational differentiation of the function $\Psi_{\Sigma}(\mathbf{x})$ with respect to itself at the same spatial point. Here and in what follows, when writing $\delta(\mathbf{0})$, we imply that a proper regularization like the split point or a lattice one has been applied in order to make sense of this singular expression. This is the regularization which is usually implied when the second variational derivative is used in the functional Schrödinger equation in QFT.

The time derivative $\mathrm{i} \partial_{t} \Psi_{\Sigma}(\mathbf{x}, t)$ is determined by the restriction of precanonical Schrödinger equation (1.3) to $\Sigma$, which takes the form

$$
\mathrm{i} \partial_{t} \Psi_{\Sigma}=-\mathrm{i} \alpha^{i}\left(\frac{d}{d x^{i}}-\partial_{i} A_{\mu}^{a}(\mathbf{x}) \frac{\partial}{\partial A_{\mu}^{a}}\right) \Psi_{\Sigma}+\beta \frac{1}{\varkappa} \widehat{H}_{\Sigma} \Psi_{\Sigma},
$$

where $\frac{d}{d x^{i}}$ is the total derivative along $\Sigma$ :

$$
\frac{d}{d x^{i}}:=\partial_{i}+\partial_{i} A_{\mu}^{a}(\mathbf{x}) \frac{\partial}{\partial A_{\mu}^{a}}+\partial_{i} A_{\mu, k}^{a}(\mathbf{x}) \frac{\partial}{\partial A_{\mu, k}^{a}}+\ldots
$$

$A_{\mu, k}^{a}$ denote the fiber coordinates of the first-jet bundle of the bundle of YM potentials $A_{\mu}^{a}$ over space-time (c.f. [41]) and $\widehat{H}_{\Sigma}$ is the restriction to $\Sigma$ of the operator on the right-hand side of (1.3):

$$
\frac{1}{\varkappa} \widehat{H}=\frac{1}{2} \varkappa \frac{\partial^{2}}{\partial A_{a}^{\mu} \partial A_{\mu}^{a}}-\frac{1}{2} \mathrm{i} g C_{b c}^{a} A_{\mu}^{b} A_{\nu}^{c} \gamma^{\nu} \frac{\partial}{\partial A_{\mu}^{a}},
$$

which is called the De Donder-Weyl Hamiltonian operator (of YM field) [14, 15]. As $\widehat{H}$ contains no spatial (horizontal) derivatives, its restriction $\widehat{H}_{\Sigma}=\widehat{H}$. Similarly, the restricted wave function $\Psi_{\Sigma}(\mathbf{x})$ is also required to obey the restriction of the constraints (1.4) to $\Sigma$ :

$$
\hat{\pi}_{a}^{(\mu \nu)} \Psi_{\Sigma}(\mathbf{x}) \approx 0 \Leftrightarrow\left(\gamma^{\mu} \partial_{A_{\nu}^{a}}+\gamma^{\nu} \partial_{A_{\mu}^{a}}\right) \Psi_{\Sigma}(\mathbf{x}) \approx 0
$$


Let us note here the recent discussion of the classical counterpart of this constraint in 33 .

Now, by substituting (2.5) into (2.2) and using (2.7), we obtain

$$
\begin{aligned}
\mathrm{i} \partial_{t} \Psi= & \int d \mathbf{x} \operatorname{Tr}\left\{\frac{\delta \Psi}{\delta \Psi_{\Sigma}^{T}(\mathbf{x})}(\underbrace{-\mathrm{i} \alpha^{i} \frac{d}{d x^{i}}}_{I}+\underbrace{\mathrm{i} \alpha^{i} \partial_{i} A_{j}^{a}(\mathbf{x}) \frac{\partial}{\partial A_{j}^{a}}}_{I I}+\underbrace{\mathrm{i} \alpha^{i} \partial_{i} A_{0}^{a}(\mathbf{x}) \frac{\partial}{\partial A_{0}^{a}}}_{I I I}\right. \\
& \underbrace{-\frac{1}{2} \varkappa \beta \frac{\partial}{\partial A_{i}^{a}} \frac{\partial}{\partial A_{i}^{a}}}_{I V}+\underbrace{\frac{1}{2} \varkappa \beta \frac{\partial}{\partial A_{0}^{a}} \frac{\partial}{\partial A_{0}^{a}}}_{V} \underbrace{-\frac{1}{2} \mathrm{i} g \beta C_{b c}^{a} A_{i}^{b} A_{j}^{c} \gamma^{j} \frac{\partial}{\partial A_{i}^{a}}}_{V I} \\
& \underbrace{-\frac{1}{2} \mathrm{i} g \beta C_{b c}^{a} A_{i}^{b} A_{0}^{c} \gamma^{0} \frac{\partial}{\partial A_{i}^{a}}}_{V I I} \underbrace{-\frac{1}{2} \mathrm{i} g \beta C_{b c}^{a} A_{0}^{b} A_{j}^{c} \gamma^{j} \frac{\partial}{\partial A_{0}^{a}}}_{V I I I}) \Psi_{\Sigma}(\mathbf{x})\} .
\end{aligned}
$$

Let us see if this equation can reproduce the functional derivative Schrödinger equation (1.1) in some sense.

Due to the constraint $\hat{\pi}_{a}^{(i j)} \Psi_{\Sigma} \approx 0$ in $(\underline{2.8)}$ the term $(I I)$ takes the form

$$
I I: \quad \mathrm{i} \alpha^{i} \partial_{[i} A_{j]}^{a}(\mathbf{x}) \frac{\partial}{\partial A_{j}^{a}} \Psi_{\Sigma}(\mathbf{x}) .
$$

By combining it with the term $(V I)$, we obtain

$$
\frac{\mathrm{i}}{2} \beta \gamma^{i} F_{i j}^{a} \partial_{A_{j}^{a}} \Psi_{\Sigma}(\mathbf{x})
$$

Then, using the constraint $\hat{\pi}_{a}^{(i 0)} \Psi_{\Sigma} \approx 0$, we transform the latter to the form

$$
I I+V I: \quad \frac{\mathrm{i}}{2} \gamma^{i j} F_{i j}^{a} \partial_{A_{0}^{a}} \Psi_{\Sigma}(\mathbf{x})
$$

Further, by comparing the terms $(I V)$ and $(V)$ in (2.9) with the expression for the total second variational derivative of $\boldsymbol{\Psi}$ in (2.4), we conclude that the latter can be reproduced if $\beta \varkappa$ is replaced by the (regularized value of the) $(n-1)$-dimensional Dirac delta function at equal spatial points $\delta(\mathbf{0})$ :

$$
\beta \varkappa \mapsto \delta(\mathbf{0})
$$

In this case, using (2.4) the terms $(I V)$ and $(V)$ lead to

$$
\begin{gathered}
\operatorname{Tr}\left\{\frac{\delta \Psi}{\delta \Psi_{\Sigma}^{T}(\mathbf{x}, t)} \beta \varkappa \partial_{A_{\mu}^{a}} \partial_{A_{a}^{\mu}} \Psi_{\Sigma}(\mathbf{x})\right\} \mapsto \frac{\boldsymbol{\delta}^{2} \boldsymbol{\Psi}}{\boldsymbol{\delta} A_{a}^{\mu}(\mathbf{x}) \boldsymbol{\delta} A_{\mu}^{a}(\mathbf{x})}-\frac{\bar{\delta}^{2} \mathbf{\Psi}}{\bar{\delta} A_{\nu}^{a}(\mathbf{x}) \bar{\delta} A^{a \nu}(\mathbf{x})} \\
-\operatorname{Tr} \operatorname{Tr}\left\{\frac{\delta^{2} \boldsymbol{\Psi}}{\delta \Psi_{\Sigma}^{T}(\mathbf{x}) \otimes \delta \Psi_{\Sigma}^{T}(\mathbf{x})} \partial_{A_{\mu}^{a}} \Psi_{\Sigma}(\mathbf{x}) \otimes \partial_{A_{a}^{\mu}} \Psi_{\Sigma}(\mathbf{x})\right\} \\
-2 \operatorname{Tr}\left\{\frac{\delta \bar{\delta} \mathbf{\Psi}}{\delta \Psi_{\Sigma}^{T}(\mathbf{x}) \bar{\delta} A^{a \nu}(\mathbf{x})} \partial_{A_{\nu}^{a}} \Psi_{\Sigma}(\mathbf{x})\right\}
\end{gathered}
$$


The meaning of the replacement (2.13) can be understood, e.g., with the lattice regularization in mind, when the regularized value of $\delta(\mathbf{0})$ can be expressed in terms of the lattice spacing $a$ as $a^{-n+1}$. Then one can write the regularized version of (2.13) also as $\frac{1}{\varkappa} \beta \mapsto a^{n-1}$. When $a$ is infinitesimal, the right-hand side of the latter expression becomes $\mathrm{d} \mathbf{x}$ and thus the substitution (2.13) can be seen as the inverse of the quantization map (1.6) underlying precanonical quantization. Moreover, the relation defining the delta function: $\int d \mathbf{x} \delta(\mathbf{x})=1$, can be interpreted as a definiton of the inverse of $d \mathbf{x}$ which is represented by $\frac{1}{x} \beta$ under the precanonical quantization, eq. (1.6). Hence, the inverse of $d \mathbf{x}$, a regularized delta function localized at $\mathbf{x}=\mathbf{0}$, is given by the inverse of $\frac{1}{\varkappa} \beta$ : $\beta \varkappa \mapsto \delta(\mathbf{0})$, which is exactly the singular limiting map in (2.13). Since the map (1.6), which replaces differential forms by the elements of Clifford algerba, is an essential part of precanonical quantization, its inverse in (2.13), which will be shown to be a crucial element of transition from the precanonical quantization to the functional Schrödinger representation, can be seen as a partial dequantization.

The third term on the right-hand side of (2.14) can be shown to be vanishing - under the replacement (2.13) - by the following argument. Let us notice that any new term $-U$ added to the pure Yang-Mills Lagrangian, which, e.g., may describe interactions with other fields, adds a term $+U$ to the DW Hamiltonian operator $\widehat{H}$ in (1.3) (c.f. its derivation in [14,15]) and consequently, it will add the additional term

$$
\operatorname{Tr} \int d \mathbf{x} \frac{\delta \Psi}{\delta \Psi_{\Sigma}^{T}(\mathbf{x})} \frac{1}{\varkappa} \beta U(\mathbf{x}) \Psi_{\Sigma}(\mathbf{x})
$$

on the right-hand side of (2.9). If the functional Schrödinger representation can be deduced from the precanonical description, this term should correspond to the additional term $\int d \mathbf{x} U(\mathbf{x}) \Psi$ in the canonical Hamiltonian operator acting on the Schrödinger wave functional in (1.1). Because the term $U$ can be an arbitrary function of YM fields and external fields, this correspondence can be only possible if $\operatorname{Tr}\left\{\frac{\delta \Psi}{\delta \Psi_{\Sigma}^{T}(\mathbf{x})} \frac{1}{\varkappa} \beta \Psi_{\Sigma}(\mathbf{x})\right\}$ in (2.15) reproduces $\boldsymbol{\Psi}$ for any $\mathbf{x}$, i.e. it is required that

$$
\forall \mathbf{x}: \quad \operatorname{Tr}\left\{\frac{\delta \Psi}{\delta \Psi_{\Sigma}^{T}(\mathbf{x})} \frac{1}{\varkappa} \beta \Psi_{\Sigma}(\mathbf{x})\right\} \mapsto \Psi .
$$

Hence, the dependence of the functional $\Psi$ on $\Psi_{\Sigma}(\mathbf{x})$ is identical at all points $\mathbf{x}$. This observation suggests that the functional $\Psi$ can be expressed in terms of a continuous product over all points $\mathbf{x}$ of identical expressions including $\Psi_{\Sigma}(\mathbf{x})$. In this case, however, $\operatorname{Tr}\left\{\frac{\delta \Psi}{\delta \Psi_{\Sigma}^{T}(\mathbf{x})} \Psi_{\Sigma}(\mathbf{x})\right\}=\delta(\mathbf{0}) \Psi$. Therefore, again, the map in (2.16) exists only when $\frac{1}{\varkappa} \beta \delta(\mathbf{0}) \mapsto 1$ which is equivalent to the substitution given by (2.13). Then, by acting by $\frac{\delta}{\delta \Psi_{\Sigma}(\mathbf{x})}$ on both sides of (2.16), we conclude, similarly to eq. (3.15) in [21], that the third term in (2.14) vanishes under the very same substitution (2.13):

$$
\operatorname{Tr} \operatorname{Tr}\left\{\frac{\delta^{2} \boldsymbol{\Psi}}{\delta \Psi_{\Sigma}^{T}(\mathbf{x}) \otimes \delta \Psi_{\Sigma}^{T}(\mathbf{x})} \partial_{A_{\mu}^{a}} \Psi_{\Sigma}(\mathbf{x}) \otimes \partial_{A^{a \mu}} \Psi_{\Sigma}(\mathbf{x})\right\} \mapsto 0 .
$$

Therefore, from (2.14) and (2.17) it follows that the terms $(I V)$ and $(V)$ in (2.9) under the substitution (2.13) are mapped to the following expression in variational 
derivatives of $\boldsymbol{\Psi}$ :

$$
\begin{aligned}
\operatorname{Tr}\left\{\frac{\delta \Psi}{\delta \Psi_{\Sigma}^{T}(\mathbf{x}, t)} \frac{1}{2} \beta \varkappa \partial_{A_{\mu}^{a}} \partial_{A_{a}^{\mu}} \Psi_{\Sigma}(\mathbf{x})\right\} \mapsto & \frac{1}{2} \frac{\boldsymbol{\delta}^{2} \boldsymbol{\Psi}}{\boldsymbol{\delta} A_{a}^{\mu}(\mathbf{x}) \boldsymbol{\delta} A_{\mu}^{a}(\mathbf{x})}-\frac{1}{2} \frac{\bar{\delta}^{2} \boldsymbol{\Psi}}{\bar{\delta} A_{\nu}^{a}(\mathbf{x}) \bar{\delta} A^{a \nu}(\mathbf{x})} \\
& -\operatorname{Tr}\left\{\frac{\delta \bar{\delta} \mathbf{\Psi}}{\delta \Psi_{\Sigma}^{T}(\mathbf{x}) \bar{\delta} A^{a \nu}(\mathbf{x})} \partial_{A_{\nu}^{a}} \Psi_{\Sigma}(\mathbf{x})\right\} .
\end{aligned}
$$

Our next step is to consider the terms with $\partial_{A_{0}^{a}} \Psi_{\Sigma}(\mathbf{x})$ in (2.9). Namely, (2.12) and the last term in (2.18) corresponding to $\nu=0$. Their total contribution to the functional derivative Schrödinger equation, which should not have explicit terms with $\Psi_{\Sigma}$, should be zero. Therefore, from (2.12) and the temporal part of the last term in (2.18), which is a part of the term $(\mathrm{V})$ in (2.9), we obtain

$$
\frac{\mathrm{i}}{2} \frac{\delta \Psi}{\delta \Psi_{\Sigma}^{T}(\mathbf{x})} \gamma^{i j} F_{i j}^{a}(\mathbf{x})-\frac{\delta \bar{\delta} \mathbf{\Psi}}{\delta \Psi_{\Sigma}^{T}(\mathbf{x}) \bar{\delta} A_{0}^{a}(\mathbf{x})}=0 .
$$

Introducing the notation

$$
\frac{\delta \Psi}{\delta \Psi_{\Sigma}^{T}(\mathrm{x})}=: \boldsymbol{\Phi}(\mathrm{x})
$$

where $\boldsymbol{\Phi}(\mathbf{x})$ is an $\mathbf{x}$-dependent Clifford-algebra-valued functional of $\Psi_{\Sigma}(\mathbf{x})$ and $A(\mathbf{x})$, eq. (2.19) takes the form

$$
\frac{\mathrm{i}}{2} \boldsymbol{\Phi}(\mathbf{x}) \gamma^{i j} F_{i j}^{a}(\mathbf{x})-\frac{\bar{\delta} \boldsymbol{\Phi}(\mathbf{x})}{\bar{\delta} A_{0}^{a}(\mathbf{x})}=0 .
$$

The solution $\mathbf{\Phi}(\mathbf{x})$ can be written in the form which is implying the substitution (2.13) again:

$$
\boldsymbol{\Phi}(\mathbf{x})=\left.\boldsymbol{\Xi}\left[\Psi_{\Sigma}(\mathbf{x}) ; \check{\mathbf{x}}\right] e^{\frac{\mathrm{i}}{2 \varkappa} \beta \gamma^{i j} A_{0}^{a}(\mathbf{x}) F_{i j}^{a}(\mathbf{x})}\right|_{\beta \varkappa \mapsto \delta(\mathbf{0})},
$$

where $\boldsymbol{\Xi}\left[\Psi_{\Sigma}(\mathbf{x}) ; \check{\mathbf{x}}\right]$ is a functional of $\Psi_{\Sigma}(\mathbf{x})$ on the punctured space $\mathbb{R}^{n-1} \backslash\{\mathbf{x}\}$ (c.f. eq. (3.21) in [21]) which plays here a role of the integration constant. Then the solution of (2.20) takes the form (up to a normalization factor which will also include «)

$$
\left.\boldsymbol{\Psi} \sim \operatorname{Tr}\left\{\boldsymbol{\Xi}\left[\Psi_{\Sigma}(\mathbf{x}) ; \check{\mathbf{x}}\right] e^{\frac{\mathrm{i}}{2 \varkappa} \beta \gamma^{i j} A_{0}^{a}(\mathbf{x}) F_{i j}^{a}(\mathbf{x})} \frac{\beta}{\varkappa} \Psi_{\Sigma}\left(A_{\mu}^{a}(\mathbf{x})\right)\right\}\right|_{\beta \varkappa \mapsto \delta(\mathbf{0})},
$$

where the multiplier $\frac{\beta}{\varkappa}$ enters to the left from $\Psi_{\Sigma}$ in order to compensate $\delta(\mathbf{0})$ which will appear from the variation of $\operatorname{Tr}\left\{\boldsymbol{\Phi}(\mathbf{x}) \Psi_{\Sigma}(\mathbf{x})\right\}$ with respect to $\Psi_{\Sigma}(\mathbf{x})$. Note that the solution in this form is consistent with (2.16) which was already established by means of an independent reasoning.

Since the formula (2.23) should be valid for any $\mathbf{x}$, we conclude that the wave functional $\boldsymbol{\Psi}$ has the structure of a continuous product over $\mathbf{x}$ :

$$
\left.\Psi \sim \operatorname{Tr} \prod_{\mathbf{x}}\left\{e^{\frac{\mathrm{i}}{2 \varkappa} \beta \gamma^{i j} A_{0}^{a}(\mathbf{x}) F_{i j}^{a}(\mathbf{x})} \frac{\beta}{\varkappa} \Psi_{\Sigma}\left(A_{\mu}^{a}(\mathbf{x})\right)\right\}\right|_{\beta \varkappa \mapsto \delta(\mathbf{0})}
$$


This rather symbolic expression can be understood, up to a normalization factor, as the multiple Volterra product integral over $\mathbf{x}$ [39] denoted as $\pi_{\mathbf{x}} f(\mathbf{x})^{\mathrm{dx}}$ :

$$
\left.\boldsymbol{\Psi} \sim \operatorname{Tr} \int_{\mathbf{x}}\left\{e^{\frac{\mathrm{i}}{2 \varkappa} \beta \gamma^{i j} A_{0}^{a}(\mathbf{x}) F_{i j}^{a}(\mathbf{x})} \frac{\beta}{\varkappa} \Psi_{\Sigma}\left(A_{\mu}^{a}(\mathbf{x})\right)\right\}\right|_{\frac{1}{\varkappa} \beta \mapsto \mathrm{d} \mathbf{x}} .
$$

Let us note here that the multiple product integral is very little known and explored, while the one-dimensional product integral is well known under different names such as the Peano series or "path (or time) ordered exponential" used in QFT (see also [38]). A multidimensional generalization of the "path ordering" is discussed in 39] and it is probably not unique. Nevertheless, the expression (2.25) already serves the purpose of the paper as it shows that the description in terms of the Schrödinger wave functional, which is known to require additional constructs, such as regularization, in order to be usable within the existing QFT (see e.g. [5, 6]), emerges from the well defined precanonical description only in a limiting case when both the substitution $\frac{1}{\varkappa} \beta \mapsto \mathrm{d} \mathbf{x}$ and the multiple product integral itself have to be understood as the continuum limits of certain discretized or otherwise regularized expressions, which also require additional constructs in order to be mathematically correctly defined.

Next, after integrating by parts and using the constraint $\hat{\pi}_{a}^{(i 0)} \Psi_{\Sigma} \approx 0$, the term $(I I I)$ in (2.9) transforms to

$$
\text { III : } \quad \mathrm{i} A_{0}^{a}(\mathbf{x}) \partial_{i} \operatorname{Tr}\left(\mathbf{\Phi}(\mathbf{x}) \partial_{A_{i}^{a}} \Psi_{\Sigma}(\mathbf{x})\right) .
$$

Using the constraint $\widehat{\pi}_{a}^{(i 0)} \Psi_{\Sigma} \approx 0$ in $(V I I I)$, the of sum $(V I I)$ and (VIII) yields

$$
V I I+V I I I: \quad \mathrm{i} g C_{b c}^{a} A_{0}^{b}(\mathbf{x}) A_{i}^{c}(\mathbf{x}) \operatorname{Tr}\left\{\mathbf{\Phi}(\mathbf{x}) \partial_{A_{i}^{a}} \Psi_{\Sigma}(\mathbf{x})\right\} .
$$

Therefore, the terms $(I I I),(V I I)$ and $(V I I I)$ together lead to

$$
I I I+V I I+V I I I: \quad \mathrm{i} A_{0}^{b}(\mathbf{x}) D_{i b}^{a} \operatorname{Tr}\left\{\boldsymbol{\Phi}(\mathbf{x}) \partial_{A_{i}^{a}} \Psi_{\Sigma}(\mathbf{x})\right\}
$$

where $D_{i b}^{a}:=\delta_{b}^{a} \partial_{i}+g C_{b c}^{a} A_{i}^{c}(\mathbf{x})$ denotes the covariant derivative. By remembering the expression of the first total variational derivative in (2.3), we see that the terms $(I I I),(V I I)$ and $(V I I I)$ in (2.9) yield

$$
\mathrm{i} \int d \mathbf{x} A_{0}^{b}(\mathbf{x})\left(\delta_{b}^{a} \partial_{i}+g C_{b c}^{a} A_{i}^{c}(\mathbf{x})\right)\left(\frac{\delta \Psi}{\delta A_{i}^{a}(\mathbf{x})}-\frac{\bar{\delta} \boldsymbol{\Psi}}{\bar{\delta} A_{i}^{a}(\mathbf{x})}\right),
$$

that resembles the standard appearance of the quantum Gauß constraint in the canonical Hamiltonian operator of YM theory (see e.g. [40]).

Now, let us consider the total derivative in term $(I)$ in (2.9). By integration by parts and using the cyclic permutation under the trace, we obtain

$$
-\int d \mathbf{x} \operatorname{Tr}\left\{\frac{\delta \Psi}{\delta \Psi_{\Sigma}^{T}(\mathbf{x})} \alpha^{i} \frac{d}{d x^{i}} \Psi_{\Sigma}(\mathbf{x})\right\}=\int d \mathbf{x} \operatorname{Tr}\left\{\frac{d}{d x^{i}} \mathbf{\Phi}(\mathbf{x}) \alpha^{i} \Psi_{\Sigma}(\mathbf{x})\right\} .
$$

Using (2.22), on the right-hand side of (2.30) we have

$$
\left.\int d \mathbf{x} \operatorname{Tr}\left\{\mathbf{\Phi}(\mathbf{x}) \frac{d}{d x^{i}}\left(-\frac{\mathrm{i}}{2 \varkappa} \beta \gamma^{k l} A_{0}^{a}(\mathbf{x}) F_{k l}^{a}(\mathbf{x})\right) \alpha^{i} \Psi_{\Sigma}(\mathbf{x})\right\}\right|_{\beta \varkappa \mapsto \delta(\mathbf{0})} .
$$


By the cyclicity of trace it equals to

$$
\begin{aligned}
& \left.\int d \mathbf{x} \operatorname{Tr}\left\{\Psi_{\Sigma}(\mathbf{x}) \mathbf{\Phi}(\mathbf{x}) \frac{d}{d x^{i}}\left(-\frac{\mathrm{i}}{2 \varkappa} \beta \gamma^{k l} A_{0}^{a}(\mathbf{x}) F_{k l}^{a}(\mathbf{x}) \alpha^{i}\right)\right\}\right|_{\beta \varkappa \mapsto \delta(\mathbf{0})} \\
& =\left.\operatorname{Tr}\left\{\beta\|\boldsymbol{\Psi}\| \int d \mathbf{x} \frac{d}{d x^{i}}\left(-\frac{\mathrm{i}}{2} \gamma^{k l} A_{0}^{a}(\mathbf{x}) F_{k l}^{a}(\mathbf{x}) \gamma^{i}\right)\right\}\right|_{\beta \varkappa \mapsto \delta(\mathbf{0})}=0,
\end{aligned}
$$

where $\|\Psi\|:=\frac{1}{\varkappa} \beta \Psi_{\Sigma}(\mathbf{x}) \boldsymbol{\Phi}(\mathbf{x})$ is the matrix-valued functional whose trace is $\boldsymbol{\Psi}$ (c.f. (2.22), (2.23) ), hence $\mathrm{x}$-independent. The integral on the right-hand side of (2.32) vanishes as its integrand is a total divergence. Therefore, we have shown that the term $(I)$ in (2.9) does not contribute to the functional derivative equation for $\boldsymbol{\Psi}$ (again, under the limiting map (2.13)):

$$
I: \quad \int d \mathbf{x} \operatorname{Tr}\left\{\frac{\delta \Psi}{\delta \Psi_{\Sigma}^{T}(\mathbf{x})} \alpha^{i} \frac{d}{d x^{i}} \Psi_{\Sigma}(\mathbf{x})\right\} \mapsto 0 .
$$

Our next step is to consider the $\partial_{A_{0}^{a}} \partial_{A_{a}^{0}} \Psi_{\Sigma}(\mathbf{x})$ term $(V)$ in (2.9). To this end, let us recall that the behavior of $\Psi_{\Sigma}(\mathbf{x})$ is determined by the precanonical Schrödinger equation restricted to $\Sigma$, eq. (2.5). Our analysis above has shown that the total derivative term in (2.5) has no contribution to the functional Schrödinger equation and that some other terms in (2.9) play together to produce the Gauß constraint (c.f (2.29)). Hence the part of the time evolution of $\Psi_{\Sigma}(\mathbf{x})$ which is relevant for the time evolution of the Schrödinger wave functional $\Psi$ is controlled by the remaining terms inside the round brackets in (2.9) (c.f. (2.12)):

$$
\mathrm{i} \partial_{t} \Psi_{\Sigma} \approx \beta\left(\frac{\mathrm{i}}{2} \beta \gamma^{i j} F_{i j}^{a}(\mathbf{x}) \partial_{A_{0}^{a}}+\frac{\varkappa}{2} \partial_{A_{0}^{a} A_{0}^{a}}-\frac{\varkappa}{2} \partial_{A_{i}^{a} A_{i}^{a}}\right) \Psi_{\Sigma} .
$$

This equation can be seen as the restriction of precanonical Schrödinger equation (1.3) to $\Sigma$ and the subspace of constraints given by (2.8) and the initial value (Gauß) constraint. By writing the r.h.s. of (2.34) in the form of a "magnetic Schrödinger operator" with a constant Clifford-valued "magnetic potential" in the space of temporal components $A_{0}^{a}$, i.e.

$$
\mathrm{i} \partial_{t} \Psi_{\Sigma}=\beta\left(-\frac{1}{2}\left(\mathrm{i} \sqrt{\varkappa} \partial_{A_{0}^{a}}-\frac{1}{2 \sqrt{\varkappa}} \beta \gamma^{i j} F_{i j}^{a}(\mathbf{x})\right)^{2}-\frac{1}{4 \varkappa} F_{i j}^{a}(\mathbf{x}) F^{a i j}(\mathbf{x})-\frac{\varkappa}{2} \partial_{A_{i}^{a} A_{i}^{a}}\right) \Psi_{\Sigma},
$$

we conclude that the dependence of $\Psi_{\Sigma}$ on $A_{0}^{a}(\mathbf{x})$ can be absorbed in the phase factor:

$$
\Psi_{\Sigma}\left(A_{0}^{a}(\mathbf{x}), A_{i}^{a}(\mathbf{x}), t\right)=(1+\beta) e^{-\frac{i}{2 \varkappa} \beta \gamma^{i j} A_{0}^{a}(\mathbf{x}) F_{i j}^{a}(\mathbf{x})} \Phi_{\Sigma}\left(A_{i}^{a}(\mathbf{x}), t\right),
$$

where the projecting factor $(1+\beta)$ is present because of the identity $\beta(1+\beta)=1+\beta$. Then

$$
\frac{1}{2} \beta \varkappa \partial_{A_{0}^{a} A_{0}^{a}} \Psi_{\Sigma}(\mathbf{x})=\frac{1}{2} \beta \varkappa\left(\frac{-\mathrm{i} \beta}{2 \varkappa} \gamma^{i j} F_{i j}^{a}\right)^{2} \Psi_{\Sigma}(\mathbf{x})=\frac{\beta}{4 \varkappa} F_{i j}^{a} F_{a}^{i j} \Psi_{\Sigma}(\mathbf{x}),
$$


where the identity $\left(\gamma^{i j} F_{i j}\right)^{2}=-2 F_{i j} F^{i j}$ is used. Therefore, by taking into account (2.16), the term $(V)$ in (2.9) under the limiting map (2.13) yields:

$$
V: \quad \operatorname{Tr} \int \mathrm{d} \mathbf{x}\left\{\boldsymbol{\Phi}(\mathbf{x}) \frac{1}{2} \beta \varkappa \partial_{A_{0}^{a} A_{0}^{a}} \Psi_{\Sigma}(\mathbf{x})\right\} \mapsto \frac{1}{4} F_{i j} F^{i j} \Psi
$$

Thus we see that the right hand side of (2.38) correctly reproduces the magnetic energy term in (1.1).

Further, from (2.16) and (2.36) we obtain, up to a normalization factor,

$$
\forall \mathbf{x}:\left.\quad \boldsymbol{\Psi} \sim \operatorname{Tr}\left\{\boldsymbol{\Phi}(\mathbf{x}) \frac{1}{\varkappa}(1+\beta) e^{-\frac{\mathrm{i}}{2 \varkappa} \beta \gamma^{i j}} A_{0}^{a}(\mathbf{x}) F_{i j}^{a}(\mathbf{x}) \Phi_{\Sigma}\left(A_{i}^{a}(\mathbf{x}), t\right)\right\}\right|_{\beta \varkappa \mapsto \delta(\mathbf{0})} .
$$

By comparison with the form of $\mathbf{\Phi}(\mathbf{x})$ obtained in (2.22), we conclude that the phase factors with $A_{0}^{a}$ in $\mathbf{\Phi}(\mathbf{x})$ and $\Psi_{\Sigma}(\mathbf{x})$ actually cancel each other at each point $\mathbf{x}$, so that

$$
\forall \mathbf{x}:\left.\quad \boldsymbol{\Psi} \sim \operatorname{Tr}\left\{\boldsymbol{\Xi}(\check{\mathbf{x}}) \frac{1}{\varkappa}(1+\beta) \Phi_{\Sigma}\left(A_{i}^{a}(\mathbf{x}), t\right)\right\}\right|_{\beta \varkappa \mapsto \delta(\mathbf{0})} .
$$

This again suggests that (up to a normalization factor which may include $\varkappa$ ) the functional $\boldsymbol{\Psi}$ is expressed as the product integral of wave functions $(1+\beta) \Phi_{\Sigma}(\mathbf{x})$ :

$$
\left.\boldsymbol{\Psi} \sim \operatorname{Tr} \int_{\mathbf{x}}\left\{(1+\beta) \Phi_{\Sigma}\left(A_{i}^{a}(\mathbf{x}), t\right)\right\}\right|_{\frac{\beta}{\varkappa} \mapsto \mathrm{d} \mathbf{x}} .
$$

From this expression it is obvious that the partial variational derivatives of $\boldsymbol{\Psi}\left[\Phi_{\Sigma}\left(A_{i}^{a}(\mathbf{x}), t\right)\right]$ with respect to $A_{i}^{a}(\mathbf{x})$ are actually vanishing:

$$
\frac{\bar{\delta} \mathbf{\Psi}}{\bar{\delta} A_{i}^{a}(\mathbf{x})}=0
$$

Then the term $(I V)$ in (2.9) produces just the second variational derivative of $\boldsymbol{\Psi}$ (c.f. $(2.18)$ ):

$$
I V: \quad-\operatorname{Tr} \int \mathrm{d} \mathbf{x}\left\{\boldsymbol{\Phi}(\mathbf{x}) \frac{1}{2} \beta \varkappa \partial_{A_{i}^{a} A_{i}^{a}} \Psi_{\Sigma}(\mathbf{x})\right\} \mapsto-\frac{1}{2} \frac{\delta^{2} \mathbf{\Psi}}{\delta A_{i}^{a}(\mathbf{x})^{2}},
$$

and the last term in (2.29) is vanishing, so that the terms $(I I I),(V I I)$ and $(V I I I)$ exactly reproduce the usual Gauß constraint term in the canonical Hamiltonian operator:

$$
I I I+V I I+V I I I: \quad \mathrm{i} \int d \mathbf{x} A_{0}^{b}(\mathbf{x}) D_{i b}^{a} \frac{\delta \Psi}{\delta A_{i}^{a}(\mathbf{x})} .
$$

Summarizing the results of the above consideration of (2.9), we have shown that

- the terms $(I I I),(V I I)$ and $(V I I I)$ together with the antisymmetry constraint (2.8) reproduce the Gauß constraint term in the canonical Hamiltonian operator,

- the term $(I V)$ reproduces the second variational derivative term in the canonical Hamiltonian operator in the limiting case $\beta \varkappa \mapsto \delta(\mathbf{0})$, 
- the terms $(I I),(V)$ and $(V I)$ together with the antisymmetry constraint (2.8) allow us to obtain a product integral formula relating the Schrödinger wave functional and the precanonical wave function, and to reproduce the correct magnetic contribution $\frac{1}{4} F_{i j}^{a} F_{a}^{i j}$ to the Yang-Mills Hamiltonian operator in the temporal gauge, 1$]$

- the term $(I)$ does not contribute to the Hamiltonian operator (assuming the precanonical wave function is vanishing at the spatial infinity).

By combining those results we conclude that, in the limiting case (2.13), we obtain from the precanonical Schrödinger equation (1.3) and precanonical quantum constraints (1.4) the following equation on the functional $\Psi$ :

$$
\mathrm{i} \partial_{t} \Psi=\int \mathrm{d} \mathbf{x}\left(-\frac{1}{2} \frac{\delta^{2}}{\delta A_{i}^{a}(\mathbf{x})^{2}}+\frac{1}{4} F_{i j}(\mathbf{x}) F^{i j}(\mathbf{x})+\mathrm{i} A_{0}^{a}(\mathbf{x}) D_{i a}^{b} \frac{\delta}{\delta A_{i}^{b}(\mathbf{x})}\right) \Psi .
$$

The temporal components $A_{0}^{a}$ appear here as the Lagrange multipliers which fix the quantum version of the Gauß constraint (1.2). The rest of the equation is just the canonical Schrödinger equation for quantum Yang-Mills field (1.1) which one usually derives using the canonical quantization in the temporal gauge (see e.g. [8,40]).

Thus, in the limiting case when $\beta \varkappa$ goes to (a regularized value of) $\delta(\mathbf{0})$ (i.e. essentially to the UV cutoff of the momentum space volume), we have derived from the precanonical Schrödinger equation (1.3) and the quantum antisymmetry constraints (1.4) the canonical functional derivative Schrödinger equation (1.1) in the temporal gauge, the Gauß constraint (1.2), and the explicit product integral formula (2.25) relating the Schrödinger wave functional with the Clifford-valued precanonical wave function.

\section{Conclusion}

We have shown in our previous papers that the approach of precanonical quantization leads to the description of quantum pure Yang-Mills theory in terms of the Cliffordalgebra-valued precanonical wave functions on the space of Yang-Mills field components $A_{\mu}^{a}$ and space-time coordinates $x^{\mu}$. The precanonical wave function satisfies the analogue of the Schrödinger equation defined on the aforementioned bundle, eq. (1.3), which treats all space-time dimensions on equal footing. The expressions of precanonical quantum operators typically contain an ultraviolet parameter $\varkappa$ of the dimension of an inverse spatial volume.

This description contrasts with the more familiar description of quantum YM fields in terms of the Schrödinger wave functionals of field configurations $A_{\mu}^{a}(\mathbf{x})$ at fixed moments of time $t$ which is derived from canonical quantization and implies a distinction between the space variable $\mathbf{x}$ and the time variable $t[8,40]$.

In this paper, we investigated a connection between the description of quantum YM theory based on precanonical quantization and the standard description in the functional Schrödinger representation. We demonstrated that the latter can be derived from the

\footnotetext{
${ }^{1}$ Note that in our previous paper 14, where the temporal gauge was imposed by hand, we actually obtained a wrong result $\frac{1}{8} F^{2}$ for the magnetic energy, see the erroneous transition from (4.17) and (4.20) to eq. (4.21).
} 
former in the limiting case of an infinitesimal value of $1 / \varkappa$ when the Clifford algebra element $\gamma_{0} / \varkappa$ can be mapped to the differential form representing an infinitesimal volume element $\mathrm{dx}$. In this singular limiting case, we were able to derive both the standard functional derivate Schrödinger equation for the quantum YM field and the Gauss law constraint from the precanonical Schrödinger equation, and also to obtain an expression of the Schrödinger wave functional of quantum YM theory in terms of a multiple product integral of precanonical wave functions restricted to a field configuration $A_{\mu}^{a}=A_{\mu}^{a}(\mathbf{x})$.

Our results suggest that the standard functional Schrödinger representation of quantum field theory of Yang-Mills fields appears from the precanonical formulation as a singular limiting case. While the former, in order to be a well-defined theory at least on the physical level of rigour, is known to require an ad hoc regularization (e.g. a point split in the second variational derivative in the functional derivative Schrödinger equation (1.1)), which typically introduces a UV cutoff scale $\Lambda$ as a necessary additional element of the theory removed by a subsequent renormalization, the precanonical formulation is "already regularized" by introducing the ultraviolet scale $\varkappa$ as an inherent element of the precanonical quantization procedure.

One should notice that the introduction of the ultraviolet scale $\varkappa$ in precanonical quantization does not introduce a grainy structure of space-time at ultra-small scales or violate the relativistic space-time at the corresponding high energies. In the case of quantum mechanics, which corresponds to one-dimensional space-time, $\varkappa$ is dimensionless and the Clifford-algebra-valued precanonical wave function is complex valued, hence a usual quantum mechanics is reproduced. The question still remains if $\varkappa$ is a universal fundamental scale or it is an auxiliary element of precanonical quantization of fields which should be removed from the physical results by a procedure similar to the usual renormalization.

Quite unexpectedly, our recent estimations of the scale of $\varkappa$ based on the estimation of the cosmological constant in precanonical quantum gravity [29] and the estimation of the YM mass gap [37] have consistently pointed to the possible subnuclear scale of $\varkappa$, which contradicts a more plausible naive expectation that the scale of $\varkappa$ is Planckian. The physical meaning of these results is still to be explored.

Acknowlegdements: I gratefully appreciate the hospitality of the School of Physics and Astronomy of the University of St Andrews, Scotland, and 24/7 availability of its facilities for research.

\section{References}

[1] R. P. Feynman, The qualitative behavior of Yang-Mills theory in $2+1$ dimensions, Nucl. Phys. B188 (1981) 479-512.

[2] K. Gawędzki, Yang-Mills theory as Schrödinger quantum mechanics on the space of gauge-group orbits, Phys. Rev. D26 (1982) 3593-3610.

[3] G.C. Rossi, M. Testa, The structure of Yang-Mills theories in the temporal gauge I-III, Nucl. Phys. B163 (1980) 109, ibid. B176 (1980) 477, ibid. B237 (1984) 442.

[4] B. F. Hatfield, Gauge invariant regularization of Yang-Mills wave functions, Phys. Lett. 154B (1985) 296-302. 
[5] R. Jackiw, Analysis on infinite dimensional manifolds: Schrödinger representation for quantized fields, in Field Theory and Particle Physics, eds. O. Éboli, M. Gomes and A. Santoro (World Scientific, Singapore 1990) p. 78-143.

[6] M. Lüscher, R. Narayanan, P. Weisz and U. Wolff, The Schrödinger functional - a renormalizable probe for non-abelian theories, Nucl. Phys. B384 (1992) 168-228.

[7] Sung Ku Kim, Wuk Namgung, Kwang Sup Soh and Jae Hyung Yee, Equivalence between the Weyl, Coulomb, and unitary gauges in the functional Schrödinger picture, Phys. Rev. D 41 (1990) 3792-5.

Sung Ku Kim, Wuk Namgung, Kwang Sup Soh and Jae Hyung Yee, Equivalence between the covariant, Weyl, and Coulomb gauges in the functional Schrödinger picture, Phys. Rev. D 43 (1991) 2046-9.

[8] B. Hatfield, Quantum Field Theory of Point Particles and Strings, Reading, MA: Addison-Wesley (1992).

[9] P. Mansfield and M. Sampaio, Yang-Mills beta function from the large distance expansion of the Schrödinger functional, Nucl. Phys. B545 (1999) 623-655, hep-th/9807163.

[10] J.N. Islam, Schrödinger functional equation for Yang-Mills theory, Prog. Theor. Phys. 89 (1993) 161-185.

[11] J. Pachos, The Coulomb branch of Yang-Mills theory from the Schrödinger representation, Phys. Lett. B432 (1998) 187-190, hep-th/9801163.

[12] Š. Olejník, The Yang-Mills vacuum wave functional thirty-five years later, J. Phys.: Conf. Ser. 631012057 (2015), arXiv:1503.01296 [hep-lat].

[13] S. Krug, The Yang-Mills Vacuum Wave Functional in $2+1$ Dimensions, PhD Thesis, UAB, Barcelona (2014), arXiv:1404.7005 [hep-th]. See also: S. Krug and A. Pineda, The regularization and determination of the Yang-Mills vacuum wave functional in three dimensions at $O\left(e^{2}\right)$, Nucl. Phys. 878 (2014) 82-116, arXiv:1308.2663 [hep-th].

[14] I.V. Kanatchikov, Precanonical quantization of Yang-Mills fields and the functional Schrödinger representation, Rep. Math. Phys. 53 (2004) 181-193, hep-th/0301001.

[15] I.V. Kanatchikov, Ehrenfest theorem in precanonical quantization, J. Geom. Symmetry Phys. 37 (2015) 43, arXiv:1501.00480

[16] I.V. Kanatchikov, Toward the Born-Weyl quantization of fields, Int. J. Theor. Phys. 37 (1998) $333-42$, quant-ph/9712058.

[17] I.V. Kanatchikov, De Donder-Weyl theory and a hypercomplex extension of quantum mechanics to field theory, Rep. Math. Phys. 43 (1999) 157-70, hep-th/9810165.

[18] I.V. Kanatchikov, On quantization of field theories in polymomentum variables, in: Particles, Fields and Gravitation, (Proc. Int. Conf. Łódź, Poland, Apr. 1998) ed. J. Rembielinski AIP Conf. Proc. vol. 453, p. 356-67, Amer. Inst. Phys., Woodbury (NY) 1998, hep-th/9811016.

[19] I.V. Kanatchikov, Geometric (pre)quantization in the polysymplectic approach to field theory, hep-th/0112263.

[20] I.V. Kanatchikov, Precanonical quantization and the Schrödinger wave functional revisited, Adv. Theor. Math. Phys. 18 (2014) 1249-65, arXiv:1112.5801 [hep-th].

[21] I.V. Kanatchikov, On the precanonical structure of the Schrödinger wave functional, Adv. Theor. Math. Phys. 20 (2016) 1377-96, arXiv:1312.4518 [hep-th].

[22] I.V. Kanatchikov, Precanonical quantization and the Schrödinger wave functional, Phys. Lett. A283 (2001) 25-36, hep-th/0012084. 
[23] Th. De Donder, Théorie Invariantive du Calcul des Variations, Gauthier-Villars, Paris (1935); H. Weyl, Geodesic fields in the calculus of variations, Ann. Math. (2) 36 (1935) 607-29.

H. Rund, The Hamilton-Jacobi Theory in the Calculus of Variations, D. van Nostrand, Toronto (1966).

H. Kastrup, Canonical theories of Lagrangian dynamical systems in physics, Phys. Rep. 101 (1983) $1-167$.

[24] I.V. Kanatchikov, Canonical structure of classical field theory in the polymomentum phase space, Rep. Math. Phys. 41 (1998) 49-90, hep-th/9709229.

I.V. Kanatchikov, On field theoretic generalizations of a Poisson algebra, Rep. Math. Phys. 40 (1997) 225-34, hep-th/9710069

[25] F. Hélein and J. Kouneiher, The notion of observable in the covariant Hamiltonian formalism for the calculus of variations with several variables, Adv. Theor. Math. Phys. 8 (2004) 735-777, math-ph/0401047.

F. Hélein and J. Kouneiher, Covariant Hamiltonian formalism for the calculus of variations with several variables, Adv. Theor. Math. Phys. 8 (2004) 565-601, math-ph/0211046.

[26] M. Forger, C. Paufler and H. Römer, The Poisson bracket for Poisson forms in multisymplectic field theory, Rev. Math. Phys. 15 (2003) 705, math-ph/0202043.

[27] I. Kanatchikov, On a generalization of the Dirac bracket in the De Donder-Weyl Hamiltonian formalism, in Differential Geometry and its Applications, eds. Kowalski O., Krupka D., Krupková O. and Slovák J. (World Scientific, Singapore, 2008) pp. 615-625. arXiv:0807.3127.

[28] I.V. Kanatchikov, Precanonical quantum gravity: quantization without the space-time decomposition, Int. J. Theor. Phys. 40 (2001) 1121-49, gr-qc/0012074.

See also: I.V. Kanatchikov, gr-qc/9810076, gr-qc/9912094, gr-qc/0004066

[29] Igor V. Kanatchikov, On the "spin-connection foam" picture of quantum gravity from precanonical quantization, The Fourteenth Marcel Grossmann Meeting on Recent Developments in Theoretical and Experimental General Relativity, Astrophysics, and Relativistic Field Theories, Part D, Ed. M. Bianchi, R.T. Jantzen and R. Ruffini (World Scientific, Singapore, 2018) 3907-3915, arXiv:1512.09137 [gr-qc].

Igor V. Kanatchikov, Ehrenfest theorem in precanonical quantization of fields and gravity, The Fourteenth Marcel Grossmann Meeting on Recent Developments in Theoretical and Experimental General Relativity, Astrophysics, and Relativistic Field Theories, Part C, Ed. M. Bianchi, R.T. Jantzen and R. Ruffini (World Scientific, Singapore, 2018) 2828-2835, arXiv:1602.01083 [gr-qc].

I.V. Kanatchikov, On precanonical quantization of gravity in spin connection variables, AIP Conf. Proc. 1514 (2012) 73-76, arXiv:1212.6963 [gr-qc].

I.V. Kanatchikov, De Donder-Weyl Hamiltonian formulation and precanonical quantization of vielbein gravity, J. Phys. Conf. Ser. 442, 012041 (2013), arXiv:1302.2610 [gr-qc].

I.V. Kanatchikov, On precanonical quantization of gravity, Nonlin. Phenom. Complex Sys. (NPCS) 17 (2014), 372-376, arXiv:1407.3101 [gr-qc].

[30] M. Castrillón López and J.E. Marsden, Some remarks on Lagrangian and Poisson reduction for field theories, J. Geom. Phys. 48 (2003) 52-83.

[31] R. Cianci, S. Vignolo and D. Bruno, Geometrical aspects in Yang-Mills gauge theories, J. Phys. A37 (2004) 2519-26.

[32] F. Hélein, Multisymplectic formulation of Yang-Mills equations and Ehresmann connections, arXiv:1406.3641 [math-ph].

[33] J. Berra-Montiel, E. del Río and A. Molgado, Polysymplectic formulation for topologically massive Yang-Mills field theory, Int. J. Mod. Phys. A32 1750101 (2017), arXiv:1702.03076. 
[34] J. Berra-Montiel, A. Molgado and D. Serrano-Blanco, De Donder-Weyl Hamiltonian formalism of MacDowell-Mansouri gravity, Class. Quant. Grav. 34235002 (2017).

[35] J. Gaset and N. Román-Roy, Multisymplectic unified formalism for Einstein-Hilbert gravity, J. Math. Phys. 59032502 (2018).

J. Gaset and N. Román-Roy, New multisymplectic approach to the metric-affine (Einstein-Palatini) action for gravity, arXiv: 1804.06181.

[36] D. Vey, Multisymplectic formulation of vielbein gravity: I. De Donder-Weyl formulation, Hamiltonian ( $n-1)$-forms, Class. Quant. Grav. 32 (2015) 095005, arXiv:1404.3546 [math-ph].

F. Hélein and D. Vey, Curved space-times by crystallization of liquid fiber bundles, Found. Phys. 47 (2017) 1-41, arXiv:1508.07765 [math-ph].

[37] Igor V. Kanatchikov, On the spectrum of DW Hamiltonian of quantum SU(2) gauge field, Int. J. Geom. Meth. Mod. Phys. 14 (2017) 1750123, arXiv:1706.01766 [hep-th].

[38] R.L. Karp, F. Mansouri and J.S. Rno, Product integral representations of Wilson lines and Wilson loops, and Non-Abelian Stokes theorem, Turk. J. Phys. 24 (2000) 365-84, hep-th/9903221.

R.L. Karp, F. Mansouri and J.S. Rno, Product integral formalism and non-Abelian Stokes theorem, J. Math. Phys. 40 (1999) 6033-43, hep-th/9910173

[39] V. Volterra and B. Hostinský, Opérations Infinitésimales Linéaires, (Gauthier-Villars, Paris, 1938). A. Slavík, Product Integration, its History and Applications, (Matfyzpress, Prague, 2007).

[40] K. Huang, Quarks, Leptons and Gauge Fields, (World Scientific, Singapore, 1992).

[41] D.J. Saunders, The Geometry of Jet Bundles, (Cambridge University Press, Cambridge, 1989). 